\title{
A Computational Model for Arguments Understanding
}

\author{
Stéphane Guez ( $\left.{ }^{*}\right)$ \\ Department of Computer Science \\ University of Rochester \\ Rochester, NY 14627 (USA)
}

\section{Introduction}

This paper presents a computational model for the understanding of arguments in natural language dialogues. Previous work on argumentation in Artificial Intelligence has been mainly focused on the conceptual aspects. But argumentation is also a linguistic phenomenon. Language provides structures to express arguments, to orient the argumentative interpretation of utterances, or to present a new concept or a new piece of information as an argument in favor of or against a certain conclusion, independently of the actual contents of these propositions. Argumentation also affects the discourse structure. The order in which arguments can be uttered is constrained.

The purpose of this paper is to describe the different aspects of argumentation, and to propose a model which integrates the different levels of analysis of argumentative phenomena: the conceptual level, the linguistic level, and the discourse level.

\section{What is Argumentation?}

The study of argumentation involves the understanding of the propositional content of utterances, as well as the analysis of their linguistic structure, the relations with the preceding and following utterances, the recognition of the underlying conceptual beliefs, and general understanding within the global coherence of the discourse. Argumentative analysis relies on several sources of knowledge: linguistic constraints, domain dependent conceptual relations, and discourse structure. None of them is sufficient by itself for a complete analysis, but they all contribute to it, especially if one source of information is incomplete, for instance if the beliefs of the speaker are unknown or unusual or if the semantic content is ambiguous. In any dialogue, one of these sources may be missing, without preventing the speakers from fully understanding the statements and positions of the other participants in the conversation, providing that the speakers remain coherent in the way they organize and express their beliefs.

It is not possible to view argument understanding as a linear process, going from syntactic and semantic analysis to conceptual interpretation and global pragmatic understanding. In many cases, the very purpose of a conversation is to allow the participants

(") Current address of the author: BULL - CEDIAG, 68 Route de Versailles, 78430 Louveciennes (France). E-mail: guez@cediag.bull.fr to present their views on a subject. Therefore, the hypothesis that the other participants can rely on a complete description of the beliefs of the speakers to fully understand their arguments does not hold in real situations. But the linguistic structure of the arguments may often be analyzed independently of their content, and reveal constraints from which we can derive the information that can make up for these gaps in the knowledge about the domain and the speakers beliefs and intentions. A complete model of argument understanding must also include a model of learning. It is our intention to focus on non conceptual sources of knowledge, mainly the structural constraints which can provide essential information to understand arguments.

An argumentative analysis is aimed at understanding how the arguments relate to each other: what is said, in favor of which proposition, based on which beliefs and towards which intention.

Consider the following example, adapted from [Cohen 1987]:

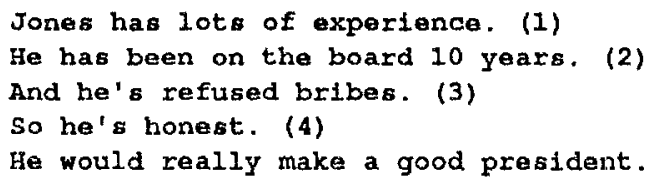

To understand this discourse is to figure out how propositions relate to and support each other:

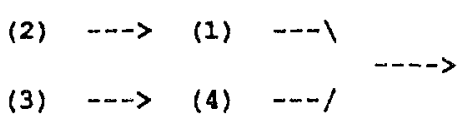

Prior conceptual knowledge about the domain, as well as the discourse properties of so and and helps to guess at once the structure of the arguments in the sequence of statements in this example. A closer analysis may also reveal that we do not really need a complete prior knowledge of all the conceptual relations involved in the example. The use of so in (4) not only informs us that the following proposition is given as conclusion of the previous one (3), and not the other way around, but also tells us that this is the only reasonable possibility. Any proposition in (4) had to be a valid conclusion for (3) and we could have learned from the entire sequence that the speaker views refusing bribes as a definite reason to declare someone honest.

Consider now the following dialogue between two speakers planning a big family reunion:

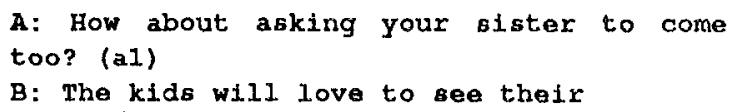


coustris. (b1)

But; it' $s$ such a long trip. (b2)

Bessides you know how $I$ f feel about my

brother-1n-1.aw. (b.3)

(b3) contains a strong semantic ambiguity, though the reader will not have any doubt about its overall meaning: it is a rejection of (a1). But how / feel may mean I don't like him as well as I like him. The interpretation must rely on contextual information and structural properties. A careful argumentative analysis reveals that the structure of the dialogue $(x$ but $Y$ besides $Z$ ) strongly constrains the interpretation of (b3) almost independently of the actual content of (b3). Let us consider two variants of B's last reply:

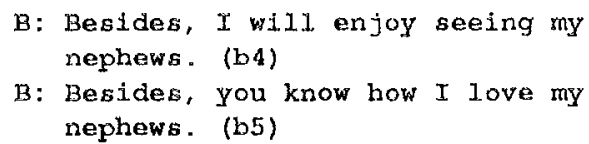

(b4) sournds like an incoherent statement, while (b5) could only be interpreted as sarcasm (how I love would just mean / hate). The linguistic structure is so strong that, whatever follows the final besides, it can only be interpreted as an argument to reject (a1) and we do not need any prior knowledge about B's beliefs to understand this dialogue.

\section{Knowledge Sources for the Analysis of Arguments}

\subsection{The Conceptual Analysis of Arguments}

Artificial Intelligence work on argumentation has been essentially focused on the conceptual level, mainly because the argumentative analysis of natural language dialogues has been generally considered as a conceptual only problem. So previous work emphasizes problems related to the logical structure of arguments and the representation of domain knowledge. Arguments are propositions supporting other propositions, and the analysis of a discourse results in a tree showing how propositions are expressed in favor of or against each other, relative to a knowledge base of basic relations and arguments.

[Flowers 1982] proposes to represent the history of a dialogue between two opponents in an argument graph and studies the strategy to generate the best next turn. The analysis is strictly conceptual and linguistic issues are mostly ignored.

Robin Cohen proposes a model for the understanding of arguments in discourse [Cohen 1984, Cohen 1987]. In her perspective, all the relations between arguments which are understood from the given text must be supported by conceptual knowledge of general or particular beliefs about what is a good argument for what. Given a sequence of statements, the question is to figure out how the propositions relate to each other. For a sequence of two related propositions, the first proposition may support the second proposition given as conclusion, or the other way around: the conclusion is given first and its justification follows. With more statements, the complexity of the computation increases dramatically as both schemes may be mixed and as more than one proposition may be expressed to support a conclusion. Sho gives an algorlthm to bullo the underlying conceptual structure incrementally which takes into account the role of clue words to limit the search about where the current sentence should be attached in the structure. The whole process relies heavily on the information provided by an "Evidence Oracle". The oracle contains a list of evidence relations, and given iwo propositions, tells whether the first one can be given as an argument for the other.

\subsection{Limits of the Conceptual Analysis}

The major difficulty raised by an exclusively conceptual treatment of argumentation is the problem of incomplete knowledge. If we do not use any other source of information or constraints about the discourse, only arguments supported by prior knowledge recorded in the knowledge base can be recognized and properly understood. Thus in such systems, the assumption is made that the complete set of beliefs of the speaker is available. We consider this assumption too strong to be fully acceptable, not only because it seems difficult to represent such a large and complex amount of knowledge, but more fundamentally because in many cases the very purpose of argumentative discourse is to present new arguments never expressed before, to reveal the beliefs and intentions of the speaker, and to present, for the first time, certain propositions as arguments in favor or against certain conclusions.

In general, incomplete knowledge about the beliefs of the speaker does not prevent the hearer from fully understanding all the arguments. In fact, new knowledge is learned while the understanding process is laking place. On the other hand, misunderstanding is a rather common phenomenon in human communication, and there is misunderstanding as soon as the speaker's discourse relies too much on knowledge which is not explicitly stated or on implicit relations which are not shared by the hearer. It should be also noted that it is common for the participants in an argumentative dialogue to intentionally use locally ambiguous formulations to express their views, while the overall orientation of their discourse is perfectly clear to the hearer.

Another major issue is whether or not to consider arguments as logical implications. To a certain extent, the natural relation "supports" shares some of the properties of the logical implication. Of course, this relation only makes sense when there is a semantic connection (or even a causal relation) between the terms, while the truth value of an implication is completely independent from the semantics of the propositions it connects. If it is acceptable to a certain extent to consider argumentative relations as logical implications and to perform logical inferences on them during the understanding process, very often it will be used for these inferences uncertain knowledge, default general relations, assumed knowledge or even relations which have just been learned and do not have a very high level of plausibility. It seems at least appropriate to be careful about a logical treatment of argumentation in a general model of argument understanding which intends to take into account other aspects of argumentative phenomenon. beyond the conceptual aspects. 


\subsection{The Linguistics of Argumentation}

The linguistic level has been relatively neglected in Al work on argumentation. Flowers, though dealing with natural language dialogues, makes almost no account of the linguistics of argumentation. If Robin Cohen proposes a linguistic analysis of the structure of argumentative discourse, she seems more concerned with discourse structure than with argumentation per se. She studies the role of clue words, but essentially their effect on the organization of discourse. This study is very interesting since it reveals many constraints imposed by the use of clue words on the order and structure of arguments. For instance, any proposition following phrases like in particular or in addition will go in the same direction as the previous part of the discourse and provide additional arguments in favor of the point delended by the speaker. The study also shows very well that there are rules about how arguments in favor of the point in case or against it can be mixed or organized into a coherent discourse. But the step Robin Cohen does not make is to truly take into account the argumentative value of clue words, in order to avoid a systematic use of the Evidence Oracle. Because of its perspective, her work sometimes ignores properties of clue words which specifically affect argumentation.

Outside of Al research, Oswald Ducrot has developed a linguistic theory of argumentation [Anscombre \& Ducrot 1983]. His concern is not to study the conceptual structure of arguments raised by two opponents in a debate, but how linguistic structures affect argumentation. His contribution to the study of argumentation is part of a larger framework, referred to as "integrated pragmatics", whose goal is to demonstrate that linguistic structures (syntax) and pragmatics must be taken into account together in the process of discourse understanding.

According to Ducrot, language provides specific structures to express arguments and constrain the discourse. Certain words orient the argumentative interpretation and the continuation of the discourse, independently of the informative content it may carry. Ducrot identifies the linguistic constraints which rule the presentation of a proposition $P$ used to make the hearer accept a conclusion $C$. It is not enough that $P$ be conceptually a good reason to accept $C$ : the linguistic structure of the utterance of $\mathrm{P}$ must also satisfy certain conditions in order for it to be, in the current discourse, an argument for $C$. For example, to say even $A$ is to present $A$ as an argument oriented towards some conclusion $\mathrm{C}$ and stronger than the arguments presented so far. It is independent of the content of $A$ and whether $A$ is really a good argument to defend the conclusion $C$.

Ducrot's work does not specifically concern clue words, but any linguistic "operator" which may affect argumentation. The argumentative features of operators that are described at the linguistic level can be viewed as constraints that affect the interpretation of utterances containing such linguistic structures. For example, to say $A$ but $B$ is to present $A$ as an argument in favor of some conclusion $C$ and to present $\mathrm{B}$ as an argument in favor of the opposite conciusion not $C$. The overall argumentative orientation of $A$ but $B$ is not $C$. This description of the use of but is independent of the actual instantiation of the argumentative variables $\mathrm{A}, \mathrm{B}$ and $\mathrm{C}$. This description takes into account the pragmatic role of but and is more general and precise than the traditional description where $A$ and $B$ are just viewed as propositions with some kind of opposition.

A very interesting point raised in Ducrot's theory is the distinction and the independence between the informational level and the argumentative level, as it is developed in [Raccah 1987]. The argumentative use of an utterance depends only partially on the informational content of this utterance. In particular, it is very often the case that while the utterance of a proposition $\mathrm{P}$ may provide very good reasons to accept a conclusion $C$, it is impossible to use $P$ in a discourse as an argument in favor of C. For instance, if the utterance You are nearly on time carries the information You are late, it cannot be used as a reproach and followed by something like You must apologize. The linguistic structure, in this case the use of nearly (it would be the same with almost), constrains a proposition to produce an argumentative effect exactly opposite to what could be expected from a strictly logical analysis of the propositional contents. Consider now the two propositions This car burns little oil and This car burns a little oil: they carry exactly the same propositional content, but if we agree with the belief that to burn oil is not a good thing for a motor, then we can very well say This car burns a little oil but the motor is in good shape, while This car burns little oil but the motor is in good shape sounds inappropriate, which can only be explained by the linguistic structure used: a combination of little or a little with but. If we assume that speakers are perfectly coherent, the latter utterance can even be interpreted as the expression of the belief that to burn oil is a good thing. Someone who really knows nothing about car mechanics would very likely interpret things that way. Argumentation is not at all exclusively determined by the conceptual relations between the content of propositions.

However, the studies of Ducrot on connectives are often long and thorough and reveal subtle aspects which go far beyond any possible reasonable formalization attempt. Raccah [Raccah 1987] made several contributions to provide a rigorous and formalized account of this work. Though he is concerned with applications within an Artificial Intelligence framework, his main goal is to define theoretical semantics of natural language. He has not try to define the role argumentation could play in an integrated computational model, and his attempts therefore cannot be articulated within a larger theory of context or a model of discourse processing.

\subsection{The Structure of Argumentative Discourse}

The structure of argumentative discourse is also constrained by the rules which apply to any discourse, and the same concepts can be used to describe it [Grosz \& Sidner 1986]. The analysis of contextual information is essential and notions such as the focus have their counterpart in argumentative dialogues: keeping track of what is currently the object of the debate contributes to the dialogue segmentation. The continuity of the point in case provides an additional criterion for the definition of a segment in argumentative discourse. 
Robin Cohen's work shows how clue words affect the order in which arguments are uttered, and she identifies rules which constrain the structure of argumentative discourse. Argumentation is essentially a relational phenomenon: how do the propositions which are uttered in a discourse relate to each other. Ducrot's work directly addresses this point: it is a study of the constraints that rule the orientation and continuation of discourse. The discourse structure provides a framework in which new propositions are attached to when they are analyzed.

\subsection{Integrating the Analysis of Arguments}

Most of the work on argumentation that we have presented is usually mainly focused on one aspect of argumentation and tends to reduce the whole problem to these aspects. From all the examples we have previously mentioned, it becomes clear that understanding arguments is not only or specirically a conceptual problem, nor is it a linguistic problem, but it is a combination of conceptual, linguistic and discourse issues that must be dealt with concurrently. An "argumentative operation" occurs when an explicit proposition is presented in favor of or against another proposition, which may remain implicit. An argumentative operation is characterized by the propositional content of the argument, the linguistic structure used to express it, and the discourse context in which it is uttered.

We view the argumentative analysis of a dialogue as the identification of the argumentative operations in this dialogue, along with the explicitation of the constraints and relations which support each operation, at the linguistic, conceptual and discourse levels. Our model then consists of several modules, each one providing an analysis which contributes to the understanding of argumentative operations. Here is a brief description of each level of analysis:

- At the Linguistic Level, the use of connectives is analyzed as constraints put on the interpretation of discourse. Knowledge consists mainly of a detailed description of the properties of linguistic structures which play an argumentative role.

- At the Conceptual Level, the conceptual structure of the arguments is analyzed. The knowledge base contains common sense relations, hierarchies of concepts and argumentative relations distributed in different belief spaces. It also describes the relative strength of arguments. This level has also the ability to make hypotheses on now beliefs and check their plausibility.

- At the Discourse Level, the discourse structure is built incrementally and the position of each utterance within the structure is recorded. This level of analysis keeps track of the argumentative focus of the discourse as well as constraints which hold through several sentences.

The essential idea is to integrate the different levels of analysis of argumentative discourse in order to come out with an interpretation which is coherent with all these levels, within each level and between them. The constraints described at the linguistic level define relation of coherence between the linguistic structure and the propositional content of utterances. We do not consider each participant in the dialogue as a perfectly logical actor, but only relations which are consistent with the current content of the bases can be hypothesized when conceptual knowledge is missing. The analysis of the discourse structure is used to maintain the global coherence of the discourse. In general, we will always consider that each participant in a dialogue only utters coherent statements.

A complete theory of argumentation must include a theory of learning. Learning new conceptual knowledge, in the course of the argumentative analysis, occurs when linguistic and discourse constraints can balance the lack of appropriate conceptual relations to interpret a particular utterance. The identification of new argumentative rules may raise conflicts between local and global coherence. Consistency with previously existing knowledge must be checked before integrating learned rules into the base. It is acceptable to conclude that a speaker does not follow a common belief, but a speaker can not contradict him/herself.

\section{A Computational Model}

Our model consists of several module. Each module contributing to the general understanding process by providing a specific set of constraints resulting from the analysis of the input:

- the Conceptual Base contains all the domain conceptual relations. It is divided in several spaces, one for general common knowledge shared by all actors, except otherwise specified, and one space for each speaker to record his/her particular beliefs.

- the Relation Finder derives appropriate relations from the conceptual knowledge represented in canonical form.

- the Base of Linguistic Constraints describes each argumentative operator.

- the Context Analyzer keeps track of the local and global topic of the conversation, the argumentative orientation of the current or previous segment, and incrementally builds the discourse structure.

- the Argumentative Analyzer actually computes the argumentative orientation of an utterance or a complete turn, taking into account the contextual constraints as well as the linguistic constraints.

- the Learning Module is activated when there is a gap in the available conceptual knowledge, resulting in the impossibility to account for the coherence of the current turn in the dialogue. This modules makes hypotheses for new relations and checks their plausibility and consistency with what is already known. The Learning Module is able to update the belief space of the current speaker.

\subsection{Representation of Conceptual Knowledge}

Conceptual knowledge is made essentially of facts and rules. Facts concern independent propositions, while rules describe argumentative relations between propositions. For instance:

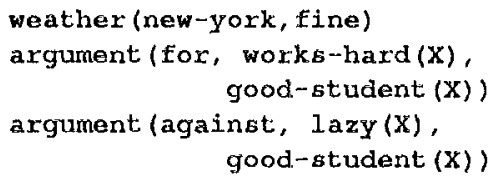


We use the operator "opposite" to consider the opposite of a proposition. This operator is not the logical negation, but we have the following rules of equivalence:

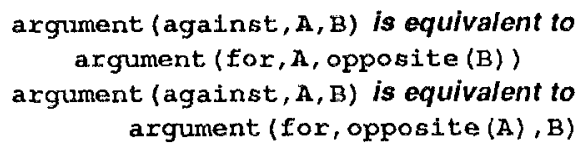

Knowledge is distributed into different belief spaces. By default, general knowledge is shared by the actors. Knowledge about semantic hierarchies is independent from belief spaces.

It is very important to insist that argumentation relations can not be assimilated to logical operators and manipulated as such. The argumentative relation "in favor of" is not processed as a logical implication. Truth values do not matter very much to interpret arguments, since we are mostly interested in the relations between propositions. In fact, the truth value of individual propositions matters all the less that in general, nothing can be logically deduced from the combinations of facts and argumentative rules.

An argumentative rule in not a description of the set of conditions that must be met for a certain conclusion to be true. A rule only defines one argument for a conclusion: it usually is a partial argument. If this argument holds, there may be at the time other arguments which hold and go against the same conclusion. This is the very source of any serious argumentative debate: opponents will raise arguments which are believed, by both, to hold, but which go in opposite directions concerning the point of the debate.

For instance, nice weather is surely a good argument to go for a walk, though it is not a sufficient condition to take such a decision. A lot of work to do is a very good argument which goes against the suggestion of a walk. Both "nice weather" and "a lot of work" can hold together, and there is no way to make any valid reasoning to conclude about going or not going for a walk. A speaker could express both facts in a discourse: what we need to understand his/her point is information about which fact is held as an argument stronger than the other.

The need for ways to compare the relative strength of arguments illustrates once again the inappropriateness of a logical model to handle the process of understanding arguments. It is the relative strength of propositions towards a certain conclusion which determines the outcome of the discourse. The predicate stronger asserts the relative strength of arguments towards the same conclusion. It takes three arguments, the two propositions to be compared and the conclusion intended by these two propositions. The predicate stronger-opp asserts the relative strength of arguments towards opposite conclusions. It takes three arguments, the two propositions to be compared and the conclusion intended by the first one (while the second proposition intends the opposite of the given conclusion). For instance:

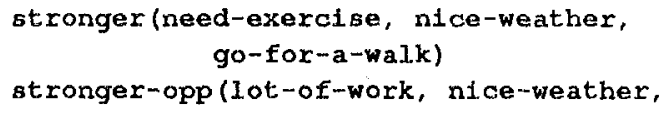

opposite (go-for-a-walk))

\subsection{Representation of Linguistic Knowledge}

Our model uses first order logic to describe relations and constraints. We represent the knowledge attached to argumentative operators as a list of local constraints which are satisfied when the operator is used. For but and almost, we have for instance:

(A but B)

$$
\begin{aligned}
& \text { argument (for, } \mathbf{A}, C \text { ) } \\
& \text { argument (against, B, C) } \\
& \text { stronger-opposite (B, A, opposite (C)) } \\
& \text { argumentative-orientation ( } \\
& \text { operator (but, } A, B \text { ), opposite (C) } \\
& \text { lmost A) } \\
& \text { argument (for, } A, C \text { ) } \\
& \text { argumentative-orientation ( } \\
& \quad \text { operator (almost, } A \text { ), C) }
\end{aligned}
$$$$
\text { operator (but, A, B), opposite (C)) }
$$

(almost A)

The predicate argumentative-orientation is used to assert the final orientation of an expression containing an operator or a connector. The orientation is given as a propositional content. The constraints which are not explicitly present when the expression is uttered are assumed to be asserted at the time of the utterance.

\subsection{Representation of Discourse Structure}

The input and output uses the same basic data structure, which is a complete description of the dialogue. The structure is augmented when constraints are taken into account and conclusions found. Descriptions use a features list format.

The dialogue is described as a hierarchy, according to the segmentation of the dialogue between turns (complete intervention of one speaker) and individual utterances. Initially, the structure only contains input information about the first utterance.

The hierarchical structure is then built incrementally. Information is added as soon as it is available, as the result of the analyses performed on the input. Within the discourse structure, at each level, the topic and the argumentative orientation are recorded.

\subsection{Algorithm for the Analysis of Arguments}

The analysis of a dialogue is performed as an incremental process. The basic algorithm consists of the following steps:

- listing the contextual constraints

- listing the linguistic constraints resulting from the use of clue words

- searching for argumentative relations coherent with the previous constraints

- computation of the argumentative orientation

It is extended to include the computation of contextual constraints and the derivation and learning of new conceptual relations. We keep track of a global topic as well as a local topic, often identified as the argumentative orientation of the current segment. An analysis is first attempted using the available concepts, and if it fails, the hypothesis mechanism is activated. Hypotheses added to a belief space can be later retracted to satisfy global 
coherence. Hypotheses may be made about missing conceptual knowledge, even in the case where these new relations are incompatible with default common knowledge, as long as this process results in a global interpretation which accounts for the coherence of the current utterances. The plausibility and consistency of new hypotheses are checked by looking for possible contradictions with existing knowlodge, interpreting for this lask argumentative relations as logical implications.

\section{Implementation and Example}

We have realized an implementation in Prolog. It is able to analyze a dialogue, io compute argumentative orieritations and to learn new conceptual relations when necessary. The syntactic/semantic analysis is not implemented. Dialogues are represented with features structures. The discourse constraints are represented as a set of rules in Prolog describing the process of any new utterance. For each operator, a se? of linguistic constraints is listed. It is a list of conditions to be satisfied to complete the processing of the current utterance.

Here is a trace of the automatic processing of the second example given in the introduction:

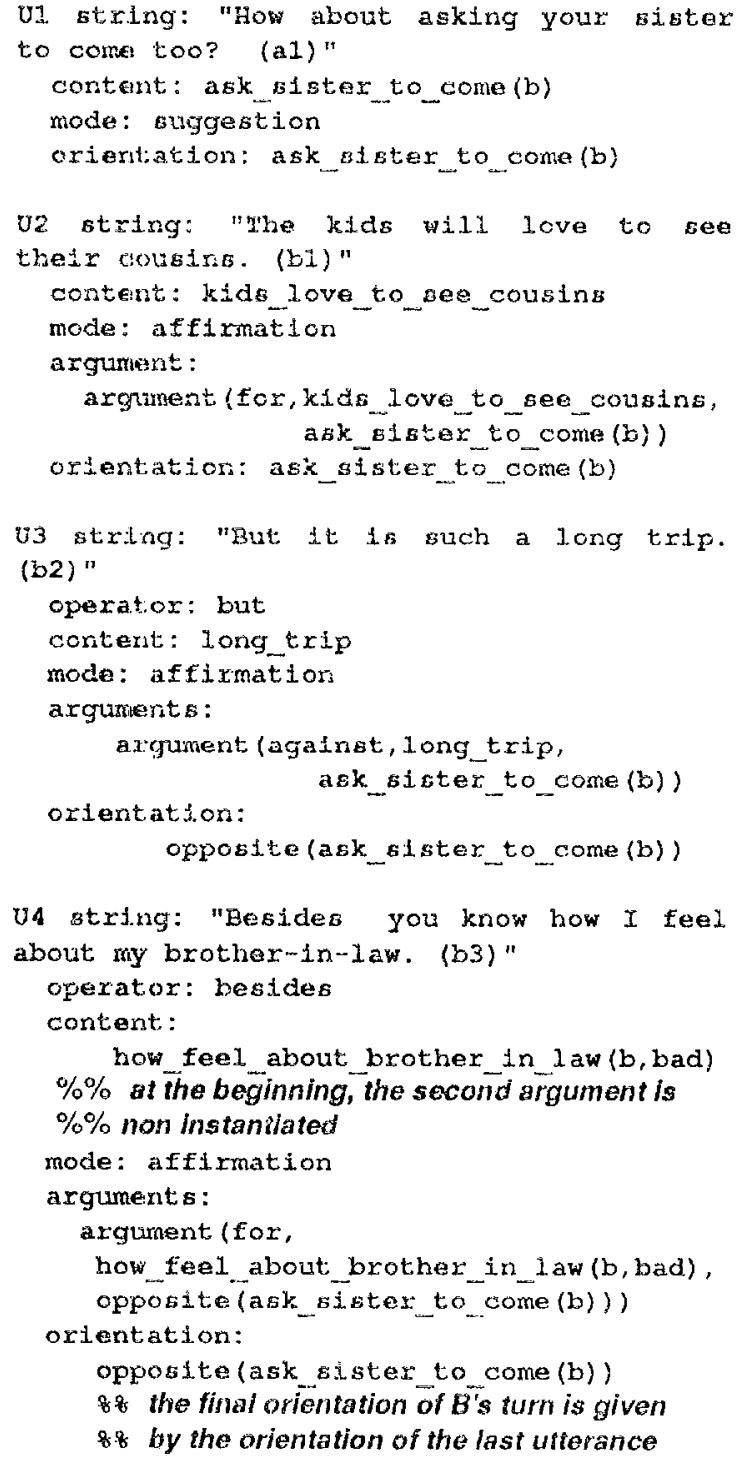

If we replace (b3) by (b3'):

B: Besides you know t feel good about xyy brother-in-lazk. (b3')

the system will finds a coherent interpretation, by making the following hypothese:

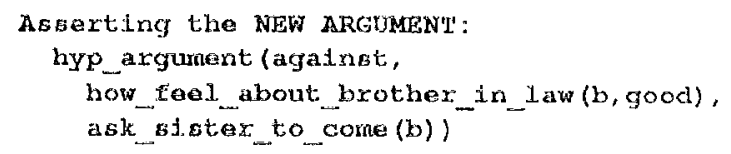

\section{Conclusion}

We have proposed a computational model which provides a more complete account of argumentation in discourse than what has been proposed before. Major clirections for future work concern improvements in the description of linguistic operators, and the iniegration within a larger model of discourse processing which would include speech acts analysis and plan recognition [Allen \& Perrault 1980]. Within this context, we believe our work to be a useful contribution to the automatic processing of natural language dialogues.

\section{References}

Allen, J. \& Perrault, $R$.

Analyzing Intentions in Uterrances.

Artificial Intelligence Journal, 15(3):143-178, 1980.

Anscombre, J.C. \& Ducrot, 0 .

L'argumentation dans la langue.

Pierre Mardaga, Bruxelles, 1983.

Cohen, R.

A Computational Theory of the Function of Clue Words in Argument Understanding.

Proceedings of COLING 84, pages 251--258, Staniord, California, July 1984.

Cohen, $\mathrm{A}$

Analyzing the Structure of Argumentative Discourse. Computational Linguistics, $13(1-2): 11-24,1987$.

Ducrot, 0 .

Les mots du discours.

Les editions de Minuit, Paris, 1980.

Flowers, M., McGuire, R. and Birnbaum, L.

Adversary Arguments and the Logic of Personal Attacks.

In Lehnert \& Ringle, editors, Strategies for Natural Langugage Processing, LEA, 1982.

Grosz, B. and Sidner, C.

Attentions, Intentions, and the Structure of Discourse.

Computational Linguistics 12(3):175-204, 1986.

Raccah, P.-Y.

Modelling Argumentation and Modelling with Argumentation.

Argumentation, Bruxelles, 1987. 